\title{
E-cigarette closed system
}

Addiction Ontology

\section{Source}

Addiction Ontology

Definition: An e-cigarette in which none of the main components is interchangeable.

Comment: These e-cigarettes are often confusingly referred to cigalikes and they have many similarities but do not need to look like cigarettes. Note that the closed 'pod' may contain either e-liquid of a salts based solution.

This definition was imported from the Addiction Ontology (see https://addictovocab.org/). Comments and suggestions for improvements are welcome using the Qeios review system.

Definitions imported from the Addiction Ontology (AddictO) are what are known as 'ontological definitions'. See this article in Qeios for an explanation https://www.qeios.com/read/YGIF9B.

Ontological definitions can sometimes be hard to read. In those cases we also include an informal definition.

Definitions also often require elaboration to make it clear how they should be used and what they include. In those cases we include a comment.

Also, definitions sometimes require an explanation as to how they came about to help users understand how they relate to alternative definitions. In those cases we include a curator note. 